\title{
A Comparative Study of Metacognitive Strategy Use between Language High Achievers and Low Achievers of English in College
}

\author{
Lihua Tang ${ }^{1}$ \\ ${ }^{1}$ School of Foreign Languages, China West Normal University, Nanchong, Sichuan, China \\ Correspondence: Lihua Tang, School of Foreign Languages, China West Normal University, Nanchong, Sichuan, \\ China. E-mail: 16867933@qq.com
}

Received: July 5, 2015 Accepted: September 14, 2015 Online Published: September 29, 2015

doi:10.5539/ijel.v5n5p147 URL: http://dx.doi.org/10.5539/ijel.v5n5p147

\begin{abstract}
Language learning is a complicated process involving a lot of individual factors; therefore, language learning effects vary greatly among individual learners. The research intends to examine the frequencies of low achievers' metacognitive strategy use in China West Normal University and to discuss whether it is necessary to propose an effective metacognitive strategy training model targeted at low achievers. The results indicate that LHAs (Language High Achievers) and LLAs (Language Low Achievers) vary considerably in metacognitive strategy use and a metacognitive strategy training program for language low achievers will be greatly needed.
\end{abstract}

Keywords: language low achievers, language high achievers, metacognitive strategies, questionnaire, training

\section{Introduction}

Metacognitive strategies are very important in Second Language Acquisition or Learning. They are executive in nature, and they are the strategies a student uses when planning, monitoring, and evaluating learning or strategy performance (Ellis, 1994). Hence, they are often referred to as self-regulatory strategies. They help learners plan、monitor and evaluate their learning process. Different studies found that what distinguished language low achievers was not the lack of appropriate strategies but the inability to choose the right strategy for the task. We take reading as examples and see in what aspects language high and low achievers differ in the use of learning strategies. In reading, first, language high achievers are aware of the general goals of reading and of the specific objectives of a particular task. In contrast, language low achievers do not tend to read for meaning. They do not adjust their reading behaviors to different kinds of content or reading situations. In addition, they do not slow down for difficult passages. Second, language high achievers are aware of and use "fix-it" strategies when problems occur. In this way, difficulties are solved before they become major problems. In contrast, language low achievers use by-pass strategies when they meet problems. Third, the strategy use of language high achievers is more flexible than that of language low achievers in two important ways. They are more likely to use different strategies in different situations, and adapt question-asking strategies to different kinds of texts. Language low achievers, in contrast, tend to apply a single strategy across contexts. Even though they may use strategies, such as context clues, they do not apply them spontaneously. Thus some researchers conclude in using learning strategies language high achievers allocate their time and effort differently for different tasks, use "fix-it" strategies when problems occur. That is to say they are more flexible in strategy use than language low achievers (Wenden, 2002).

The research intends to examine the frequencies of low achievers' metacognitive strategy use in China West Normal University and to discuss whether it is necessary to propose an effective metacognitive strategy training model targeted at low achievers. For these purposes, a comprehensive research has been carried out, in which 166 students from China West Normal University were involved. Modified SILL questionnaire was administrated to all the participants, and data were collected and analyzed using the statistical software of SPSS.

\section{Literature Review}

\subsection{A Review of Metacognitive Strategy}

Cohen (2000) define metacognitive strategies are higher order of executive skills that may entail planning for, monitoring, or evaluating the success of a learning activity. He notes that metacognitive strategies are sequential processes that one uses to control cognitive activities, and to ensure that a cognitive goal has been met. These 
processes help to regulate and oversee learning, and consist of planning and monitoring cognitive activities, as well as checking the outcome of those activities.

Nuttall (2002) regards metacognitive strategies as general skills including planning, monitoring, and evaluating, through which learners manage, direct, regulate, and guide their learning.

According to the definition of metacognitive strategies listed above, it is clear that there are similarities and agreements in these definitions. To put it simply, metacognitive strategies are skills, approaches, and thinking and actions learners use to control their cognition and learning process.

\subsection{The Relationship between Metacognitive Strategy and Other Learning Strategies}

Although scholars agree that learning strategies play an important role in improving students' performance and gradually developing independent learners, they have different opinions as to the relationship between metacognitive strategies and other learning strategies. Some claim that all the learning strategies are at the same level by supporting and connecting with each other. Others argue that metacognitive strategies are at a higher level, managing and regulating other learning strategies.

Take Oxford and O'Malley for instance. Oxford (1990) divides strategies into two main classes, direct and indirect, which are further subdivided into six groups. Indirect strategies include metacognitive strategies, social strategies and affective strategies; direct strategies include cognitive strategies, memory strategies and compensation strategies. All the six groups' strategies operate at the same level and interact with each other. But, according to O'Malley and Chamot learning strategies have been differentiated into three categories depending on the level or type of processing involved: metacognitive strategies, cognitive strategies and social and affective strategies. Metacognitive strategies are higher-order executive skills that may entail planning for, monitoring, or evaluating the success of a learning activity. They include an awareness of what one is doing and the strategies one is employing, as well as knowledge about the actual process of learning. They also include an ability to manage and regulate consciously the use of appropriate learning strategies for different situation. Obviously, O'Malley's viewpoint more complies with the definition of metacognitive strategies. The notion of metacognition was advanced as an awareness of one's own mental processes and ability to reflect on how one learns, in other words, knowing about one's knowing. Therefore, metacognitive strategies which are at a higher level play a more important role in language learning than cognitive strategies (Liu, 2010).

\section{Methodology}

\subsection{Research Questions}

The present study aims at probing into the metacognitive strategy use frequency of language low achievers in college and universities and to discuss whether it is necessary to propose an effective metacognitive strategy training model targeted at low achievers after a comparison of the strategy use between language high achievers and low achievers. For this purpose the research aims to find out the answers to the following key questions:

1. What are the differences in metacognitive strategy use between language high and low achievers?

2. Whether it is necessary to propose an effective metacognitive strategy training model targeted at low achievers.

\subsection{Subjects}

The subjects in this study consist of 166 second-year students (61 language high achievers and 105 low achievers) of non-English majors in China West Normal University for the questionnaire.

\subsection{Instruments}

There are two instruments involved in the research: Modified Strategy Inventory for Language Learning (SILL) of Oxford (1990) and CEE (College Entrance Examination).

\subsubsection{Modified SILL of Oxford}

Based on Part D of Oxford's 1990 SILL (version 5.1 and 7.0), the present questionnaire is designed with slight modifications. The adoption of SILL is due to the fact that multiple choice questions in this kind of more-structured survey can be objectively scored and analyzed so that the results are easy to summarize. Furthermore, in various versions, the SILL has been used in many parts of the world with learners of many different languages including Chinese. In order to make the questionnaire better suited to the research, the subjects are required to provide background information such as their names, majors, departments and CEE scores. Besides, the whole questionnaire is translated from its English version into Chinese so that misunderstanding of the strategy use can be reduced to the minimum. Moreover, the frequency of use of 
language learning strategy also follows Oxford's (1990) standard: "average of 4.5 to 5.0 means that the strategies are 'always or almost always used'; average of 3.5 to 4.4 means that the strategy are 'generally used'; average of 2.5 to 3.4 means that the strategies are 'sometimes used'; average of 1.5 to 2.4 means that the strategy are 'generally not used'; average of 1.0 to 1.4 means that the strategy are 'never or almost never used'; and as for the strategy use level: average of 3.5 to 5.0 means ' high level'; average of 2.5 to 3.4 means 'medium level'; average of 1.0 to 2.5 means 'low level'".

\subsubsection{CEE Scores}

CEE is used as a measurement of the subjects' language proficiency level chiefly because they are comprehensive proficiency test with high reliability and validity although oral performance is not included. The research utilizes all the 166 subjects' CEE scores of June, 2013.

\section{Results and Discussion}

\subsection{Metacognitive Strategy Use Frequency of LHAs}

Table 1. Mean and SD of LHAs' metacognitive strategy use frequency

\begin{tabular}{lll}
\hline Variables & Mean & SD \\
\hline Overall strategies & 3.32 & 0.530 \\
\hline Centering & 2.89 & 0.631 \\
Overviewing & 2.67 & 1.075 \\
Paying attention & 2.80 & 0.863 \\
Delaying speech & 3.21 & 1.180 \\
\hline Arranging and planning & 3.48 & 0.440 \\
Finding & 3.10 & 1.033 \\
Organizing & 3.65 & 0.629 \\
Setting goals & 3.11 & 0.855 \\
Identifying & 3.29 & 0.781 \\
Planning & 2.74 & 1.114 \\
Seeking practice & 3.02 & 0.650 \\
\hline Evaluating & 3.61 & 0.559 \\
Self-monitoring & 3.64 & 0.605 \\
Self-evaluating & 3.59 & 0.767 \\
\hline
\end{tabular}

Note. $\mathrm{N}=61$ persons.

As can be seen from table 1, the mean value of the overall metacognitive strategies used by LHAs is 3.32 , belonging to medium level as prescribed by Oxford (1990).

As for use of the 3 strategy groups, Evaluating with a mean of 3.61 is at the high level, followed by Arranging and planning, Centering, both of which are at the medium level.

In terms of the 11 strategy categories, the most frequently used are Organizing $(\mathrm{M}=3.65)$, Self-monitoring $(\mathrm{M}=3.64)$ and Self-evaluating $(\mathrm{M}=3.59)$, all belonging to a high level of use while the least used two are Planning with a mean of 2.74 and Overviewing with a mean of 2.67, included in the medium range.

\subsection{Metacognitive Strategy Use Frequency of LLAs}

From Table 2, it can be seen that LLAs have a low use of the overall strategies $(M=2.20)$, and compared with LHAs, the frequency is very low.

The order of the 3 strategy groups used by LLAs is ranked from the most used to the least as follows: Evaluation, Arranging and planning, Centering, all at the low level.

In terms of the 11 strategy categories, the mostly frequently used are Organizing, Self-evaluating, Finding and Delaying speech, which are at the medium level. The use frequency of Identifying $(\mathrm{M}=2.31)$, Self-monitoring $(\mathrm{M}=2.28)$, Planning $(\mathrm{M}=2.14)$, Setting goals $(\mathrm{M}=1.97)$, Paying attention $(\mathrm{M}=1.79)$, Seeking practice $(\mathrm{M}=1.78)$ and Overviewing $(\mathrm{M}=1.53)$ are all at low level. 
Table 2. Mean and SD of LLAs' metacognitive strategy use frequency

\begin{tabular}{lll}
\hline Variables & Mean & SD \\
\hline Overall strategies & 2.20 & 0.480 \\
\hline Centering & 1.95 & 0.575 \\
Overviewing & 1.53 & 0.928 \\
Paying attention & 1.79 & 0.685 \\
Delaying speech & 2.54 & 1.118 \\
\hline Arranging and planning & 2.22 & 0.557 \\
Finding & 2.54 & 1.087 \\
Organizing & 2.60 & 0.611 \\
Setting goals & 1.97 & 0.764 \\
Identifying & 2.31 & 1.064 \\
Planning & 2.14 & 1.136 \\
Seeking practice & 1.78 & 0.654 \\
\hline Evaluating & 2.42 & 0.592 \\
Self-monitoring & 2.28 & 0.749 \\
Self-evaluating & 2.57 & 0.849 \\
\hline
\end{tabular}

Note. $\mathrm{N}=105$ persons.

From the analysis, we can see: (1) Language low achievers cannot overview and link with already known material, that is to say, they cannot preview the basic principles and/or material for an upcoming language activity, and linking these with what the learners already know; (2) LLAs cannot use direct attention and selective attention comprehensively; (3) They are without aims and do not know where they are going, so they might never get the destination! Goals are generally considered to be long-range aims referring to the outcome of many months or even years. Objectives are short-term aims for hours, days, or weeks; (4) They don't know the purpose for doing something and they cannot channel their energy in the right direction; (5) They have difficulties in identifying the general nature of the task, the specific requirements of the task, the resources available within the leaner, and the need for further aids; (6) Language learners must seek out or create opportunities to practice any and all the skills. If students want to reach moderate to high proficiency, classroom time cannot usually provide adequate practice opportunities. However, LLAs do not find additional chances to practice the language although this strategy underscores students' responsibility to generate their own opportunities to practice; (7) LLAs are not used to trying to determine overgeneration from a native language rule, or inappropriate verbatim translation, helps learners understand more about the new language or about their own use of learning strategies. Therefore, according to the analysis, the strategy training program should be designed focusing on these low level used strategies.

\subsection{A Comparison of Metacognitive Strategy Use Frequency between LHAs and LLAs}

In response to the first research question, regarding the differences in metacognitive strategy use between language high and low achievers, we first look at the results of the questionnaire.

Table 3. A comparison of metacognitive strategy use frequency between LHAs and LLAs

\begin{tabular}{lllllll}
\hline Variables & Mean of $\mathrm{H}$ & $\mathrm{SD}$ of $\mathrm{H}$ & Mean of L & SD of L & $\mathrm{t}$ & $\mathrm{p}$ \\
\hline Overall strategies & 3.32 & 0.530 & 2.20 & 0.480 & 7.2449 & 0.0000 \\
\hline Centering & 2.89 & 0.631 & 1.95 & 0.575 & 4.3562 & 0.0000 \\
Overviewing & 2.67 & 1.075 & 1.53 & 0.928 & 4.2614 & 0.0000 \\
Paying attention & 2.80 & 0.863 & 1.79 & 0.685 & 3.7469 & 0.0002 \\
Delaying speech & 3.21 & 1.180 & 2.54 & 1.118 & 0.8783 & 0.3795 \\
\hline Arranging and planning & 3.48 & 0.440 & 2.22 & 0.557 & 6.3986 & 0.0000 \\
Finding & 3.10 & 1.033 & 2.54 & 1.087 & 1.226 & 0.3246 \\
Organizing & 3.11 & 0.855 & 2.60 & 0.611 & 0.9860 & 0.3255 \\
Setting goals & 3.65 & 0.629 & 1.97 & 0.764 & 6.9433 & 0.0000 \\
Identifying & 3.29 & 0.781 & 2.31 & 1.064 & 3.6444 & 0.0004 \\
Planning & 2.74 & 1.114 & 2.14 & 1.136 & 6.9433 & 0.0000 \\
Seeking practice & 3.02 & 0.650 & 1.78 & 0.654 & 5.6442 & 0.0000 \\
\hline Evaluating & 3.61 & 0.559 & 2.42 & 0.592 & 7.7480 & 0.0000 \\
Self-monitoring & 3.64 & 0.605 & 2.28 & 0.749 & 8.0668 & 0.0000 \\
Self-evaluating & 3.59 & 0.767 & 2.57 & 0.849 & 4.2097 & 0.0000 \\
\hline
\end{tabular}

Note. H represents LHAs (61 persons), U represents LLAs (105 persons). 
As is shown in Table 3, in respect of the overall strategy use, there is a statistically significant difference between LHAs and LLAs $(\mathrm{p}=0.0000)$, with the mean value of the former much higher than that of the latter. This finding shows that LHAs use the overall strategies more frequently than LLAs.

All the 3 strategy groups used by LHAs significantly different from those used by LLAs ( $p=0.0000$ in the three cases). The biggest difference lies in Evaluating ( $\mathrm{t}=7.7480)$, and then come Arranging and planning ( $\mathrm{t}=6.3986)$, and Centering ( $\mathrm{t}=4.3562)$. This result indicates that LHAs have more frequent use of the three strategy groups especially Evaluating.

Among the 11 strategy categories, LHAs and LLAs do not show any statistically significant differences in the field of Delaying speech, Finding, and Organizing; but exhibit great differences in all the other 8 strategy categories. The differences are presented here from the biggest to the smallest according to their $T$ values: Self-monitoring, Planning, Setting goals, Seeking practice, Overviewing, Self-evaluating, Paying attention, and Identifying.

\section{Practical Implications}

Through the analysis of LLAs' questionnaire a conclusion can be reached that LLAs lack strategies of Identifying, Self-monitoring, Planning, Setting goals, Paying attention, Seeking practice and Overviewing. Furthermore, considering the significant difference between LHAs and LLAs in the overall strategies, the three strategy groups and eleven strategy categories, another focus can be Identifying, Self-monitoring, Planning, Setting goals, Paying attention, Seeking practice and Overviewing.

Metacognitive strategies are a powerful tool for students both in and out of the classroom as it includes "having knowledge and having understanding, control over, and appropriating use of that knowledge." (Rubin, 2000). It is important for language low achievers to master them as the following reasons:

\subsection{Helping LLA Prepare for and Plan Their Learning}

Preparation and planning are important and can improve their learning. Students, with the help of metacognitive strategies, can set a realistic goal within a set time for accomplishing that goal. Setting clear, challenging, and realistic goals can help them see their own progress hopefully, by becoming consciously aware of their progress, the students' motivation for learning would be increased (Gourgey, 1999).

\subsection{Helping LLA Select, Use, and Orchestrate Appropriate Strategies}

The metacognitive ability to select and use particular strategies in a given context for a specific purpose means that the learner can think and make conscious decisions about the learning process (Hismanoglu, 2000). Knowing how to orchestrate the use of more than one strategy is an important metacognitive skill. The ability to coordinate, organize, and make associations among the various strategies available is a major distinction between language low and high achievers. In this study, it has shown that language high achievers tend to select strategies that work well together in a highly orchestrated way. They can easily explain the strategies they use and why they employ them.

\subsection{Helping LLA Examine and Monitor Their Strategy Use}

By examining and monitoring their use of learning strategies, language low achievers can have more chances of success in meeting their learning goals (Vann \& Abraham, 1990). They can keep themselves on track to meet their learning goals and check periodically whether or not these strategies are effective and whether or not they are still using these strategies as intended.

\subsection{Helping LLA Achieve Learner Autonomy}

Most of language low achievers fail to realize they are the person who should be responsible for their study and they still expect their teachers to explain everything to them (Williams \& Burden, 2000). Metacognitive strategies can help them take charge of their study and stimulate students' potentials and make them believe they are capable of handling their study.

\section{Limitation and Suggestions}

Although efforts have been made to ensure the reliability and validity to the research, several limitations still exists and this may probably challenge the findings generated from the research.

First, the SILL questionnaire, an instrument used in the research, is a retrospective self-report inventory that mainly depends on the abilities of the participants to understand strategies and their use even though the Chinese version is administrated in the research. Second, the index of language proficiency is based on CEE scores. Despite the high reliability and validity, CEE includes no speaking skill and a very low percentage of subjective 
testing forms. So the correlations between strategy use and proficiency may be different if a more scientific and comprehensive measurement system of proficiency is constructed.

Then, with only 166 participants involved in the two periods of research respectively, the sample of the subjects is comparatively small in size, as a result of which the findings may be far from those of the population. Besides, the sample comes from only one university, which cannot be a representative of universities with their own characteristics. Therefore, the possibility of generalizing the results in different educational environments may be limited.

Considering the limitations mentioned above and the implications discussed, some suggestions are offered here for future metacognitive strategy research and training.

As for the design of a survey, in order to achieve a high reliability, the following measures should be taken: involving a sample of large size, employing a variety of strategy assessment techniques (e.g. observation, interviews, think-aloud, note-taking, diaries, journals), and more comprehensive measurement system of proficiency. Then, the replications of this study to check its findings are encouraged so that the research can carry not only theoretical but also practical values.

All in all, it is sincerely hoped that the research findings and the suggestions will contribute to the research on metacognitive strategies and hence the improvement of English learning in colleges and universities.

\section{References}

Cohen, A. D. (2000). Strategies in learning and using a second language. Harlow, Essex: Longman.

Gourgey, A. (1999). Metacognition in basic skills instruction. Instructional Science.

Hismanoglu, M. (2000). Language Learning Strategies in Foreign Language Learning and Teaching. The Internet TESL Journal, 18.

Liu, R. Q. (2010). What can we learn from the good language learner. Foreign Language World, (2).

Nuttall, C. (2002). Teaching Reading Skills In a Foreign Language. Shanghai: Shanghai Foreign Teaching Press.

Oxford, R. L. (1990). Language learning strategies: What every teacher should know. Newbury House/Harper Collins, NY.

Rubin. (2000). Special Education for the Twenty-First Century. TESOL Quarterly, 9.

Vann, R., \& Abraham, R. (1990). Strategies of unsuccessful language learners. TESOL Quarterly, 24(2). http://dx.doi.org/10.2307/3586898

Wenden, A. (2002). Learner development in language learning. Applied Linguistics, 23, 32-55. http://dx.doi.org/10.1093/applin/23.1.32

Williams, M., \& Burden, R. L. (2000). Psychology for language teachers. Beijing: Foreign Language Teaching and Research Press.

\section{Copyrights}

Copyright for this article is retained by the author(s), with first publication rights granted to the journal.

This is an open-access article distributed under the terms and conditions of the Creative Commons Attribution license (http://creativecommons.org/licenses/by/3.0/). 